\title{
Factores asociados al inicio de la actividad sexual en adolescentes de Tuluá, Colombia
}

\author{
Yuri Paulin Holguín M. ${ }^{1}$, Luis Alfonso Mendoza T.1,2, Claudia Marcela Esquivel T. ${ }^{1}$, \\ Ricardo Sánchez M. ${ }^{1}$, Andrés Felipe Daraviña B. ${ }^{1}$, María Acuña P. ${ }^{1}$ \\ ${ }^{1}$ Unidad Central del Valle, Tuluá, Colombia. ${ }^{2}$ División de Epidemiología Hospitalaria y Unidad de Cuidados Intensivos \\ Neonatales, Fundación Hospital San José de Buga.
}

\section{RESUMEN}

Objetivo: Determinar la prevalencia de actividad sexual (AS) y factores asociados del debut sexual en adolescentes escolarizados de una ciudad colombiana. Métodos: Estudio observacional, de corte transversal, con 947 estudiantes de 14 a 19 años. Las asociaciones se determinaron mediante Odds Ratio con 95\% de intervalo de confianza. Para el análisis multivariado se empleó regresión logística. Resultados: La prevalencia de AS fue 52,8\%. La mediana de edad de inicio fue 15 años (RI: 14-16). Las variables asociadas al inicio temprano de la AS para el nivel individual fueron: sexo masculino, aprobación y actitudes permisivas hacia el sexo del adolescente, consumo de licor, tabaco y drogas. Para el nivel familiar fueron: madre no da educación sexual al hijo, hermano/a que en la adolescencia hayan sido padre/madre, aprobación y actitudes permisivas de los padres hacia el sexo en la adolescencia. Para el nivel socio-cultural fueron: como factores de riesgo el pertenecer a los estratos socioeconómicos 1 y 2, aprobación y actitudes permisivas de los pares o grupo de amigos del adolescente a la AS en la adolescencia, y como factor protector el haber recibido educación en anticoncepción por personas diferentes a los padres, como equipos de educación en colegios o personal de salud. Conclusión: Factores individuales como sexo masculino, propia toma de decisiones, papel que desempeña la madre en la educación sexual de sus hijos y la influencia de pares o amigos en la toma de decisiones, se asociaron con el inicio temprano de la AS entre adolescentes.

\section{PALABRAS CLAVE: Adolescencia, actividad sexual, alcohol, drogas psicoactivas, anticoncepción}

\section{SUMMARY}

Objective: To determine the prevalence of sexual activity (SA), age of onset and factors associated with the onset of the SA in adolescent students of a Colombian city. Methods: An observational, cross-sectional, with 947 students from 14 to 19 years old. Associations were determined using Odds Ratio with confidence interval of $95 \%$. For multivariate analysis logistic regression was used. Results: The prevalence of SA was $52.8 \%$. The median age of onset of SA was 15 years (RI: 14-16). The variables associated with early onset of SA for the individual were male approval and permissive attitudes toward sex by adolescent alcohol consumption, snuff and illicit drugs. For the family level, were the mother gives sex education to his children, a brother/daughter that in adolescence has been a parent and approval and permissive attitudes toward sex parents in adolescence. For the socio-cultural, socio-economic strata belong to 1 and 2, approval and permissive attitudes couple or group of friends to the AS teenager in adolescence, as risk factors and received education in contraception for persons other than parents and education teams in schools or health care as a protective factor, were associated with the onset of SA in adolescence. Conclusions: Individual factors 
such as male gender, one's decision-making, the role of the mother in the sex education of their children and the influence of peers and friends in decision making were found associated with early onset AS among adolescents.

\section{KEYWORDS: Adolescents, sexual activity, alcohol, illegal drugs, contraception}

\section{INTRODUCCIÓN}

La adolescencia es una etapa compleja en los seres humanos. Es un despertar en todos los ámbitos existentes y trae consigo una serie de cambios a nivel emocional, físico, hormonal y psicológico. Es esta etapa en la cual el niño alcanza su madurez física, psicológica, afectiva, intelectual y social (1).

El inicio de la actividad sexual (AS) cada vez ocurre a más temprana edad, registrando promedios de edad de inicio de 13,5 a 16 años $(2,3)$. Este inicio tan precoz de la AS, aumenta el riesgo de tener un número mayor de parejas sexuales y con ello sus riesgos (enfermedades de transmisión sexual, embarazos no deseados) (4).

Las repercusiones que tiene el inicio temprano de AS, ha generado gran interés por establecer los factores que determinan la edad de la primera relación sexual por parte de varias disciplinas, particularmente de las ciencias sociales y de la salud (5), centrándose en identificar factores del contexto social y familiar que se asocian con el comportamiento sexual de los adolescentes (sexo, nivel socio-económico, grupo étnico, estructura familiar, entre otros), mientras otros han evaluado factores psicológicos e individuales, de los cuales se sabe poco $(6,7)$, cómo es la autoestima.

Los objetivos de este trabajo fueron determinar la prevalencia de la AS, conocer la edad en la cual se inicia la AS e identificar los factores asociados al debut sexual en adolescentes escolares de colegíos públicos del Municipio de Tuluá, Colombia, para el año 2012.

\section{PACIENTES Y MÉTODOS}

La información para esta investigación fue recolectada como parte de un estudio observacional y de corte transversal, de los factores asociados al inicio de la AS en adolescentes escolarizados de noveno, décimo y undécimo grados, con edades entre 14 y 19 años de los colegios públicos de la ciudad Tuluá, Colombia, para el año 2012.

En el estudio se incluyeron adolescentes con edades entre 14 y 19 años, que se encontraran matriculados y cursando sus estudios, de ambos sexos, de cualquier estrato socioeconómico, cuyos rectores o directores hayan aprobado la realización de las encuestas, aceptado la participación de sus estudiantes en la investigación y que hubiesen firmado consentimiento informado para ser incluidos en el trabajo. Se excluyeron adolescentes escolarizados que no fueran de la ciudad de Tuluá y que padecieran alguna discapacidad.

La población de adolescentes escolares participantes fue de 6614. El tamaño de la muestra se obtuvo mediante el programa estadístico Epidat 3.1. Para hallar la prevalencia, si estimamos que el $50 \%$ de los adolescentes ha iniciado la actividad sexual, con un nivel de confianza de $95 \%$, efecto de diseño de $1 \%$ y error absoluto de $3 \%$, el tamaño de la muestra sería de 919 personas. Si asumimos que el $50 \%$ de los estudiantes de $9^{\circ}, 10^{\circ}$ y $11^{\circ}$ grado ya había iniciado la actividad sexual, para determinar una asociación estadística entre factor de riesgo y AS, con un OR de 1,4 y un IC95\%, con un poder estadístico de $80 \%$, se necesitarían 940 estudiantes. Dado que los colegios se encuentran distribuidos por toda el área urbana de la ciudad y tienen diferentes cantidades de estudiantes, se escogieron 8 de los 18 colegios con mayor volumen de estudiantes (colegios con 450 y 900 estudiantes entre los grados $9^{\circ}, 10^{\circ}$ y $11^{\circ}$ ) y que fueran representativos de todas las comunas y barrios de la ciudad. La cantidad de estudiantes de cada colegio se realizó mediante un muestreo aleatorio estratificado por afijación proporcional, y para la selección de los participantes de cada salón de 9우 $10^{\circ}$ y $11^{\circ}$ de cada colegio se empleó un muestreo aleatorio sistemático.

La variable de resultado para esta investigación fue la actividad sexual en los adolescentes (definido como coito con penetración vaginal o anal). Para cada adolescente se estudiaron variables demográficas como edad, sexo, estrato socioeconómico, grado escolar, persona con quien vive, participación dentro de la familia (hijo único o con hermanos, lugar ocupado entre los hermanos, mayor, medio o menor), nivel de escolaridad de la madre y el padre, convivencia con los padres, tipo de relación con padres y hermanos (buena o mala), consumo de alcohol, tabaco o droga psicoactiva, primera relación sexual bajo efectos de alcohol u otra droga psicoactiva, número de parejas sexuales en el último año, anticoncepción, tipo de métodos anticonceptivos, uso de condón, edad de la menarca, edad de la primera relación sexual, número de embarazos y número de hijos. Los datos fueron obtenidos mediante una encuesta estructurada autoadministrada y respondida por cada adolescente de 
manera individual bajo la supervisión y asesoría de dos de los investigadores. Esta encuesta cumplió con los requisitos de confiabilidad, validez y objetividad y fue aplicada en una prueba piloto a un grupo de estudiantes adolescentes de otro colegio diferente donde se realizó el trabajo.

Para evitar sesgos de información, a los estudiantes, docentes y rector de cada institución educativa se explicó los alcances y objetivos de la investigación, sus procedimientos y los resultados que se esperaban. Igualmente, se les indicó los beneficios que traerá para ellos, las familias y la comunidad. A su vez, se les aseguro preservar la identidad y reserva de toda la información suministrada por ellos. Las encuestas no llevaban el nombre del encuestado y se manejaron códigos que sólo el estudiante conocía y se encontraban registrados en una lista bajo custodia. Por otro lado, los investigadores desconocían al momento de la encuesta entre los adolescentes, quienes habían iniciado la actividad sexual y cuáles eran sus características.

Para evitar sesgos de selección, la cantidad de estudiantes de cada colegio a participar en la investigación se realizó mediante un muestreo aleatorio estratificado por afijación proporcional de los 8 colegios incluidos en el estudio y para la selección de los participantes de cada salón de $9^{\circ}, 10^{\circ}$ y $11^{\circ}$ de cada colegio se empleó un muestreo aleatorio sistemático.

Análisis estadístico: Los datos fueron importados desde Microsoft Office Excel 2007 al paquete estadístico STATA® 11.0 donde fueron procesados. Las variables cuantitativas se describieron de acuerdo con su distribución con promedios y desviación estándar o medianas y rangos intercuartiles. Para comparar diferencias se empleó la prueba t-test no pareado o Prueba de Mann-Whitney según correspondiera. Las variables categóricas se expresaron en frecuencias y proporciones y fueron comparadas usando la prueba de Chi2 de Pearson o prueba Exacta de Fisher. Algunas variables se agruparon para su análisis. Para determinar la asociación entre cada variable independiente y la variable resultado, se calculó el Odds Ratio (OR) con su intervalo de confianza del 95\% (IC95\%). En el análisis de las variables y la determinación de las asociaciones, las variables se dividieron en tres niveles: nivel individual, nivel familiar y nivel sociocultural de los adolescentes y sus padres. El análisis multivariado mediante regresión logística fue realizado para cada uno de los tres niveles. Se considero un valor de $\mathrm{p}<0,05$ como estadísticamente significativo.

El trabajo fue aprobado por el Comité de Ética de la Unidad Central del Valle y el Secretario de Educación y los Rectores de los colegios dieron su consentimiento para la realización de esta investigación.

\section{RESULTADOS}

Un total de 947 estudiantes fueron incluidos en el estudio, $330(34,8 \%)$ del grado 9 으 $315(33,3 \%)$ del grado $10^{\circ}$ y $302(31,9 \%)$ del grado $11 \%$ (Tabla I). El promedio de edad de los encuestados fue 16,2 \pm 1,2 años, con $389(41,1 \%)$ varones y $558(58,9 \%)$ mujeres.

Al momento del estudio, 500 (52,8\%) adolescentes ya había iniciado la actividad sexual, entre los cuales $139(42,1 \%)$ pertenecían al grado 9ㅜㅜ 171 $(54,3 \%)$ al grado $10^{\circ}$ y $190(62,9 \%)$ al grado $11^{\circ}$ (Tabla I). La mediana de la edad de inicio de la AS fue 15 años (RI: 14-16 años). Los varones (n: 263; $67,6 \%$ ) iniciaron su actividad sexual a una mediana de edad de 14 años (RI: 13-15 años) y las mujeres (n: 237; 42,5\%) 15 años (RI: 14-16 años) ( $p<0,001)$. Hallamos que entre todos los adolescentes, 180 (19\%) habían iniciado la AS antes de los 15 años, $119(30,6 \%)$ entre los varones y $61(10,9 \%)$ entre las mujeres.

El 45,8\% (n: 229) de los adolescentes que había iniciado la actividad sexual reportaron tener compañera o compañero sexual en la actualidad, 194 $(84,7 \%)$ novio/a, 11 (4,8\%) esposo/a, 18 (7,9\%) familiar y $6(2,6 \%)$ otro. Entre los adolescentes con actividad sexual, $119(23,8 \%)$ reportaron tener más de una pareja sexual al año, 89 de 263 varones (34\%) y 30 de 237 mujeres $(12,7 \%)$. Los hombres tienen una mediana de 3 (Rl: 2-3; mínimo 0 y máximo 10) parejas sexuales al año y las mujeres una mediana de 2 (RI: 0-2; mínimo 0 y máximo 5) parejas al año. La mediana de la edad de la primera pareja sexual entre los varones fue 15 años (RI: 14-16 años; mínimo 10 y máximo 28 años) y para las mujeres 18 años (RI: 16-20 años; mínimo 12 y máximo 32 años). El 27,4\% (n: 65) de las mujeres tuvieron su primera relación con una persona de 20 a 32 años, mientras el 3,4\% (n: 9) de los varones tuvieron su primera relación con personas de 20 a 28 años. Para los varones su primera pareja sexual fue amiga 111 (42,2\%), novia 122 (46,4\%), familiar $16(6,1 \%)$ y otro $14(5,3 \%)$. Para las mujeres su primera pareja sexual fue novio 199 (84\%), amigo 35 $(14,8 \%)$ y otro $3(1,2 \%)$.

En cuanto a anticoncepción, 443 (88,6\%) de los adolescentes con actividad sexual usan regular o irregularmente algún métodos (condón 84,8\%, anticonceptivos inyectables $14,5 \%$, anticonceptivos orales $4,6 \%$, anticonceptivos de implante 4,1 y otros $0,3 \%$ ). Las mujeres comenzaron a usar métodos anticonceptivos a una mediana de edad de 16 años (RI: 15-16 años). En cuanto el uso de algún método anticonceptivo en todas las relaciones sexuales, $292(58,4 \%)$ respondieron afirmativamente, $141(53,5 \%)$ varones y $151(63,8 \%)$ mujeres. El condón masculino fue usado siempre en 
Tabla I

CARACTERÍSTICAS INDIVIDUALES DE LOS ADOLESCENTES

\begin{tabular}{|c|c|c|c|c|}
\hline Características individuales & Medida & Sin AS (n: 447) & Con AS (n: 500) & $p$ \\
\hline Edad (años) & Mediana (RI) & $16(15-16)$ & $17(16-17)$ & $<0,001$ \\
\hline Sexo masculino & n (\%) & $127(28,4 \%)$ & $263(52,6 \%)$ & $<0,001$ \\
\hline \multicolumn{5}{|l|}{ Escolaridad } \\
\hline $9^{\circ}$ grado & $\mathrm{n}(\%)$ & $191(42,7 \%)$ & $139(27,8 \%)$ & $<0,001$ \\
\hline $10^{\circ}$ grado & $\mathrm{n}(\%)$ & $144(32,2 \%)$ & $171(34,2 \%)$ & \\
\hline $11^{\circ}$ grado & n (\%) & $112(25,1 \%)$ & $190(38 \%)$ & \\
\hline Edad de menarca (años) & Promedio (DE) & $12,2( \pm 1,2)$ & $12,2( \pm 1,4)$ & 0,098 \\
\hline Consumo de licor & n (\%) & $265 / 385(68,8 \%)$ & $407 / 454(89,7 \%)$ & $<0,001$ \\
\hline Consumo de tabaco & $\mathrm{n}(\%)$ & $32 / 388(8,3 \%)$ & $150 / 452(33,2 \%)$ & $<0,001$ \\
\hline Consumo de drogas & n (\%) & $10 / 383(2,6 \%)$ & $86 / 452(19 \%)$ & $<0,001$ \\
\hline $\begin{array}{l}\text { Primera relación sexual bajo } \\
\text { efecto de droga o alcohol }\end{array}$ & n (\%) & NA & $19 / 458(4,1 \%)$ & NA \\
\hline
\end{tabular}

RI: Rango intercuartil. DE: Desviación estándar. AS: Actividad sexual.

todas las relaciones sexuales en el $51,1 \%$ por varones y $50,5 \%$ de las parejas masculinas en el caso de las mujeres adolescentes, a veces en $44,9 \%$ por varones y $38,3 \%$ por mujeres y nunca en $4 \%$ por varones y $11,2 \%$ por mujeres. En el $67,5 \%$ (n: 639) de los adolescentes, la madre habló con ellos de temas relacionados con anticoncepción, mientras el padre lo hizo en $27,2 \%$ ( $n: 258$ ), sin diferencia entre los que habían y no habían iniciado la actividad sexual. En el 29,8\% (n: 132) de los adolescentes con actividad sexual, el método anticonceptivo era suministrado por la madre y en el $14,6 \%$ (n: 65) por los padres.

El 7,2\% ( $\mathrm{n}: 17$ ) de la mujeres con actividad sexual ha estado alguna vez embarazada, mientras $0,8 \%(n: 2)$ varones y $4,2 \%(n: 10)$ mujeres tienen un hijo actualmente. El $86 \%$ (n: 814) de los adolescentes reconoce que el embarazo en la adolescencia puede traer problemas tanto para la madre como para el hijo, 81,9\% (n: 366) de los que no había iniciado la actividad sexual y $90 \%$ (n: 450) de los que la habían iniciado, diferencia estadísticamente significativa $(p=0,003)$. Entre esos problemas reconocidos por los adolescentes están los económicos (n: $154 ; 18,9 \%$ ), emocionales ( $n: 75 ; 9,2 \%)$, de salud (n: $59 ; 7,2 \%$ ), escolares (n: $26 ; 3,2 \%$ ), laborales (n: 13; 1,6\%), combinación de varios tipos de problemas (n: 487; 59,8\%).

Al comparar adolescentes con y sin actividad sexual, además de hallar que los adolescentes con AS eran un año mayores (con AS, 17 años; RI: 1617 años; sin AS, 16 años; RI: 15-16 años) y en su mayoría de sexo masculino (hombres, 52,4\%; mujeres, $28 \%$ ), encontramos que los adolescentes con
AS tuvieron una mayor proporción de consumo de licor (consumo de licor, 89,7\%; no consumían licor, $68,8 \%$ ), de tabaco (fumadores, $33,3 \%$; no fumadores, $8,3 \%$ ) y drogas psicoactivas (consumidores, 19\%; no consumidores, 2,6\%), diferencias estadísticamente significativas (Tabla I). En el $4,1 \%$ de los adolescentes la actividad sexual fue iniciada bajo efectos de alcohol o drogas psicoactivas (Tabla I). Los adolescentes con AS tuvieron un hermano/a que había sido padre o madre antes de los 20 años de edad y pertenecían en su mayoría a estratos socioeconómicos 1 y 2 (estratos 1 y 2, 72,8\%; estratos mayores que 2, 63,3\%), diferencias estadísticamente significativas cuando se compara con aquellos sin actividad sexual $(p<0,001)$ (Tabla II y III). No encontramos diferencias estadísticamente significativas entre adolescentes con y sin actividad sexual, para edad de la menarca, edad, educación y ocupación de los padres, número de personas que habitaban en la casa del adolescente, ni en el aspecto de quien dependen económicamente (Tablas I, II y III).

El tiempo libre de los estudiantes adolescentes es empleado en actividades como deporte ( $n$ : 195; $20,6 \%$ ), internet (n: 94; 9,9\%), televisión (n: 65; $6,9 \%$ ), dormir (n: 46; 4,9\%), lectura (n: $20 ; 2,1 \%$ ), salir a bailar (n: $13 ; 1,4 \%)$, otras actividades ( $n$ : $111 ; 11,7 \%)$, combinación de varias actividades ( $\mathrm{n}$ : 403; 42,6\%), sin hallar diferencias estadísticamente significativa entre los adolescentes sin y con AS $(p>0,05)$. Cuando evaluamos el uso de tiempo libre en televisión y el internet en adolescentes sin AS y con AS, encontramos que no hubo diferencia estadísticamente significativa (sin AS n: 182; 40,7\%; con actividad sexual $n$ : 205; 41\%; $p=0,524$ ). 
Tabla II

\section{CARACTERÍSTICAS DE LAS FAMILIAS DE LOS ADOLESCENTES}

\begin{tabular}{|c|c|c|c|c|}
\hline Características familiares & Medida & $\operatorname{Sin}$ AS (n: 447) & Con AS (n: 500) & $p$ \\
\hline Edad de la madre & Promedio (DE) & $42,1( \pm 6,9)$ & $41,5( \pm 6,4)$ & 0,067 \\
\hline Edad de padre & Promedio (DE) & $46,4( \pm 8)$ & $46( \pm 8,4)$ & 0,663 \\
\hline Número de hermanos & Mediana (RI) & $2(1-3)$ & $2(2-3)$ & 0,006 \\
\hline Años de vida de hermano mayor & Promedio (DE) & $22,7( \pm 5,2)$ & $23,5( \pm 5,4)$ & 0,032 \\
\hline $\begin{array}{l}\text { Hermano (a) que en la adolescencia haya } \\
\text { sido padre o madre }\end{array}$ & $\mathrm{n}(\%)$ & $60(13,4 \%)$ & $116(23,2 \%)$ & $<0,001$ \\
\hline Número de personas con las que habita & Promedio (DE) & $4,3( \pm 2,3)$ & $4,4( \pm 2,3)$ & 0,523 \\
\hline
\end{tabular}

RI: Rango intercuartil. DE: Desviación estándar. AS: Actividad sexual.

Tabla III

CARACTERÍSTICAS SOCIOCULTURALES DE LAS FAMILIAS Y LOS ADOLESCENTES

\begin{tabular}{|c|c|c|c|c|}
\hline Características socioculturales & Medida & Sin AS (n: 447) & Con AS (n: 500) & $p$ \\
\hline Estrato socioeconómico & & & & 0,002 \\
\hline 1 y 2 & $\mathrm{n}(\%)$ & $283(63,3 \%)$ & $364(72,8 \%)$ & \\
\hline 3 y más & $\mathrm{n}(\%)$ & $164(36,7 \%)$ & $136(27,2 \%)$ & \\
\hline Educación de la madre & & & & 0,317 \\
\hline Primaria & n (\%) & $136(35,4 \%)$ & $146(36 \%)$ & \\
\hline Secundaria & $\mathrm{n}(\%)$ & $218(56,8 \%)$ & $239(58,9 \%)$ & \\
\hline Técnica o universitaria & n (\%) & $30(7,8 \%)$ & $21(5,1 \%)$ & \\
\hline Educación del padre & & & & 0,413 \\
\hline Primaria & n (\%) & $114(34,4 \%)$ & $110(33,3 \%)$ & \\
\hline Secundaria & $\mathrm{n}(\%)$ & $190(57,4 \%)$ & $201(60,9 \%)$ & \\
\hline Técnica o universitaria & $\mathrm{n}(\%)$ & $27(8,2 \%)$ & $19(5,8 \%)$ & \\
\hline Ocupación de la madre & & & & 0,352 \\
\hline Empleada & n (\%) & $108(24,2 \%)$ & $142(28,4 \%)$ & \\
\hline Trabajadora independiente & n (\%) & $95(21,3 \%)$ & $97(19,4 \%)$ & \\
\hline Ama de casa & $\mathrm{n}(\%)$ & $236(52,7 \%)$ & $249(49,8 \%)$ & \\
\hline Desempleada & n (\%) & $8(1,8 \%)$ & $12(2,4 \%)$ & \\
\hline Ocupación del padre & & & & 0,346 \\
\hline Empleado & n (\%) & $215(48,1 \%)$ & $255(51 \%)$ & \\
\hline Trabajador independiente & $\mathrm{n}(\%)$ & $208(46,5 \%)$ & $224(44,8 \%)$ & \\
\hline Pensionado & $\mathrm{n}(\%)$ & $24(5,4 \%)$ & $18(3,6 \%)$ & \\
\hline Desempleado & n (\%) & 0 & $3(0,6 \%)$ & \\
\hline Dependencia económica & & & & 0,234 \\
\hline Padres & n (\%) & $427(95,5 \%)$ & $462(92,4 \%)$ & \\
\hline Abuelos & $\mathrm{n}(\%)$ & $6(1,3 \%)$ & $9(1,8 \%)$ & \\
\hline Hermanos & n (\%) & $4(0,9 \%)$ & $5(1 \%)$ & \\
\hline Tíos & $\mathrm{n}(\%)$ & $2(0,45 \%)$ & $10(2 \%)$ & \\
\hline Otros & $\mathrm{n}(\%)$ & $8(1,8 \%)$ & $14(2,8 \%)$ & \\
\hline
\end{tabular}

AS: Actividad sexual. 
Para las variables del nivel individual, encontramos asociación estadísticamente significativa entre actividad sexual en la adolescencia y sexo masculino (OR: 2,8; IC 95\% 2,1-3,8), aprobación y actitudes permisivas por parte del adolescente de relaciones sexuales antes de los 20 años de edad (OR: 4,1 ; IC 95\% 3-5,8), consumo por el adolescente de licor (OR: 3,9; IC 95\% 2,7-5,8), tabaco (OR: 5,5; IC $95 \% 3,6-8,6)$ y drogas psicoactivas (marihuana, cocaína, popper, bazuco, éxtasis u otra) (OR: 8,8; IC 95\% 4,4-19,2) (Tabla IV).

Con las variables del nivel familiar, encontramos asociación estadísticamente significativa entre AS iniciada en la adolescencia y no vivir con los padres (OR; 2; IC 95\% 1,2-3,4), percepción del adolescentes de no compartir o compartir poco tiempo con la madre (OR: 2; IC 95\% 1,5-2,7), no recibir educación sexual por la madre (OR: 1,5; IC 95\% 1,1$2,1)$, percepción del adolescentes de no compartir o compartir poco tiempo con el padre (OR: 1,5; IC $95 \%$ 1,2-2), tener más de dos hermanos (OR: 1,7; IC 95\% 1,2-2,2), hermano/a que en la adolescencia fue padre o madre (OR: 1,9; IC 95\% 1,4-2,8) y padres con aprobación y actitudes permisivas hacia el sexo en la adolescencia (OR: 3,5; IC 95\% 2,6$4,7)$. No se halló asociación entre AS y la educación sexual por el padre ( $p>0,05)$ (Tabla V).

En cuanto a las variables del nivel socio-cultural encontramos que las variables que se hallaron asociadas con AS en la adolescencia fueron, pertenecer a los estratos 1 y 2 (OR: 1,6; IC 95\% 1,2-2,1) y pares o grupo de amigos del adolescente con aprobación y actitudes permisivas hacia el sexo en la adolescencia (OR: 6,6; IC 95\% 3,4-14,2) como factores de riesgo. Se halló que haber recibido información de anticoncepción por personas diferentes a los padres, como equipos de educación en colegios o personal de salud, fue factor protector (OR: 0,7; IC 95\% 0,41). No se halló asociación entre educación sexual por personas diferentes a los padres (equipos de educación en colegios o personal de salud, otros) y actividad sexual en la adolescencia (Tabla VI).

Después del análisis multivariado encontramos que, para el nivel individual las variables que se mantuvieron en el modelo fueron sexo masculino (OR: 2,4; IC 95\% 1,7-3,3), aprobación y actitudes permisivas por parte del adolescente hacia la actividad sexual antes de los 20 años de edad (OR: 3,1 ; IC95\% 2,1-4,5), consumo por el adolescente de licor (OR: 2,4; IC95\% 1,6-3,6), tabaco (OR: 2,6; IC95\% 1,5-4,3) y drogas psicoactivas (marihuana, cocaína, popper, bazuco, éxtasis u otra) (OR: 2,6; IC 5\%: 1,2-5,8), tabla IV. Para el nivel familiar, las variables que se mantuvieron en el modelo fueron no recibir educación sexual por la madre (OR: 1,6; IC95\% 1,1-2,5), hermano/a que en la adolescencia haya sido padre o madre (OR: 2,3; IC95\% 1,4-3,9) y padres que aprueban y tengan actitudes permisivas hacia la actividad sexual en la adolescencia (OR: 4,2; IC95\% 2,8-6,3) (Tabla V).
En el nivel socio-cultural del adolescente y la familia, las variables que se mantuvieron en el modelo fueron, pertenecer a los estratos socioeconómicos 1 y 2 (OR: 1,5; IC95\% 1,1-2), pares o grupo de amigos del adolescente que aprueban y tienen actitudes permisivas hacia las relaciones sexuales en la adolescencia (OR: 5,9; IC95\% 2,9-11,8) como factores de riesgo y haber recibido información planificación familiar por personas diferentes a los padres como equipos de educación en colegios o personal de salud (OR: 0,6; IC95\% 0,4-0,9) como factor protector (Tabla VI).

\section{DISCUSIÓN}

Los resultados de este estudio muestran que $52,8 \%$ de los adolescentes escolarizados entre los 14 y 19 años ya han iniciado su actividad sexual, siendo los varones los que inician a más temprana edad (una año antes que las mujeres) y con más frecuencia (67,6\% vs 42,5\%). Profamilia, Colombia, para el año 2005, estimó que aproximadamente el $21 \%$ de los adolescentes entre 15 y 19 años ya han iniciado su vida sexual, cifra mucho menor a la encontrada en nuestro trabajo (8), mientras en la Encuesta Nacional de Demografía y Salud, Colombia 2010, informó que entre las jóvenes que actualmente tienen entre 20 y 24 años, el 14\% tuvo su primera relación antes de cumplir 15 años, 60\% antes de cumplir 18 años y el $82 \%$ antes de cumplir 20 años (9). Otros trabajos informan prevalencias de actividad sexual en la adolescencia entre $19,3 \%$ y $38,2 \%$, que también son inferiores a la nuestra $(10,11)$.

El $88,6 \%$ de adolescentes de nuestro trabajo usaba algún método de anticoncepción, sin embargo, sólo el 58,4\% lo usaba en todas las relaciones sexuales. Un hecho realmente preocupante es que el $84,8 \%$ habían usado condón en sus relaciones sexuales, sin embargo, el $51,1 \%$ de los varones y $50,5 \%$ de las mujeres lo usaban siempre. Otros autores describen que sólo $81 \%$ ha usado un método anticonceptivo alguna vez en su vida y $10 \%$ los usa en su primera relación sexual (8).

El $86 \%$ de los adolescentes encuestados reconoce que el embarazo en la adolescencia puede traer problemas de tipo emocional, para la salud, económicos, escolares y de trabajo para la adolescente y para su hijo. Esto ha sido descrito por otros autores, para quienes las consecuencias de una sexualidad precoz y una maternidad en la adolescencia, trae problemas para el desarrollo educativo y personal, riesgos para la salud, riesgo de muerte y abandono de los hijos, así como la pérdida de población con potencial de instrucción y productividad para la sociedad $(12,13)$. Entre los problemas de salud para la madre adolescente y su hijo se describen, mayor incidencia de preeclampsia, trabajo de parto prematuro, prematuridad, infecciones bacterianas, sífilis y mayor mortalidad neonatal (13). 


\section{Tabla IV}

ANÁLISIS BIVARIADO Y MULTIVARIADO, NIVEL INDIVIDUAL

\begin{tabular}{|c|c|c|c|c|c|c|}
\hline Nivel Individual & $\begin{array}{l}\text { Sin AS } \\
(n: 447)\end{array}$ & $\begin{array}{l}\text { Con AS } \\
(n: 500)\end{array}$ & $\begin{array}{c}\text { ORc } \\
\text { (IC 95\%) }\end{array}$ & $\mathrm{p}$ & $\begin{array}{c}\text { ORa } \\
\text { (IC 95\%) }\end{array}$ & $p$ \\
\hline Hombre & 125 (28\%) & $262(52,4 \%)$ & $\begin{array}{c}2,8 \\
(2,1-3,8)\end{array}$ & $<0,001$ & $\begin{array}{c}2,4 \\
(1,7-3,3)\end{array}$ & $<0,001$ \\
\hline $\begin{array}{l}\text { Adolescente que } \\
\text { aprueba y tiene } \\
\text { actitudes permisivas } \\
\text { hacia la AS en la } \\
\text { adolescencia }\end{array}$ & $273(61,1 \%)$ & $433(86,6 \%)$ & $\begin{array}{c}4,1 \\
(3-5,8)\end{array}$ & $<0,001$ & $\begin{array}{c}3,1 \\
(2,1-4,5)\end{array}$ & $<0,001$ \\
\hline Consumo de licor & $265 / 385(68,8 \%)$ & $407 / 454(89,7 \%)$ & $(2,7-5,8)$ & $<0,001$ & $\begin{array}{c}2,4 \\
(1,6-3,6)\end{array}$ & $<0,001$ \\
\hline Consumo de tabaco & $32 / 386(8,3 \%)$ & $150 / 454(33 \%)$ & $\begin{array}{c}5,5 \\
(3,6-8,6)\end{array}$ & $<0,001$ & $\begin{array}{c}2,6 \\
(1,5-4,3)\end{array}$ & $<0,001$ \\
\hline Consumo de drogas & $10 / 383(2,6 \%)$ & $86 / 452(19 \%)$ & $\begin{array}{c}8,8 \\
(4,4-19,2)\end{array}$ & $<0,001$ & $\begin{array}{c}2,6 \\
(1,2-5,8)\end{array}$ & 0,017 \\
\hline
\end{tabular}

AS: Actividad sexual. ORc: Odds ratio crudo. ORa: Odds ratio ajustado.

\section{Tabla V}

ANÁLISIS BIVARIADO Y MULTIVARIADO, NIVEL FAMILIAR

\begin{tabular}{|c|c|c|c|c|c|c|}
\hline Nivel Familiar & $\begin{array}{l}\operatorname{Sin} A S \\
(n: 447)\end{array}$ & $\begin{array}{l}\text { Con AS } \\
(n: 500)\end{array}$ & $\begin{array}{c}\text { ORc } \\
(\text { IC } 95 \%)\end{array}$ & $p$ & $\begin{array}{c}\text { ORa } \\
(\text { IC 95\%) }\end{array}$ & $p$ \\
\hline No vivir con los padres & $27(6 \%)$ & $57(11,4 \%)$ & $\begin{array}{c}2 \\
(1,2-3,4)\end{array}$ & 0,004 & $\begin{array}{c}1,4 \\
(0,3-5,6)\end{array}$ & 0,646 \\
\hline $\begin{array}{l}\text { Percepción del adolescentes de no } \\
\text { compartir o compartir poco tiempo } \\
\text { con la madre }\end{array}$ & $96(21,5 \%)$ & 178 (35,6\%) & $\begin{array}{c}2 \\
(1,5-2,7)\end{array}$ & $<0,001$ & $\begin{array}{c}1 \\
(0,3-3,7)\end{array}$ & 0,974 \\
\hline $\begin{array}{l}\text { Madre no da educación sexual a } \\
\text { su hijo }\end{array}$ & $100(22,4 \%)$ & $153(30,6 \%)$ & $(1,1-2,1)$ & 0,004 & $(1,1-2,5)$ & 0,028 \\
\hline $\begin{array}{l}\text { Percepción del adolescentes de no } \\
\text { compartir o compartir poco tiempo } \\
\text { con el padre }\end{array}$ & 147 (32,9\%) & 215 (43\%) & $\begin{array}{c}1,5 \\
(1,2-2)\end{array}$ & 0,001 & $\begin{array}{c}1,4 \\
(0,7-2,6)\end{array}$ & 0,346 \\
\hline $\begin{array}{l}\text { Padre no da educación sexual a } \\
\text { su hijo }\end{array}$ & $143(32 \%)$ & 165 (33\%) & $\begin{array}{c}1 \\
(0,8-1,4)\end{array}$ & 0,741 & - & - \\
\hline Tener más de 2 hermanos & $134(30 \%)$ & $207(41,4 \%)$ & $\begin{array}{c}1,7 \\
(1,2-2,2)\end{array}$ & $<0,001$ & $\begin{array}{c}1,7 \\
(0,9-3,1)\end{array}$ & 0,082 \\
\hline $\begin{array}{l}\text { Hermano (a) que en la adolescencia } \\
\text { haya sido padre o madre }\end{array}$ & $60(13,4 \%)$ & $116(23,2 \%)$ & $\begin{array}{c}1,9 \\
(1,4-2,8) \\
3,5\end{array}$ & $<0,001$ & $\begin{array}{c}2,3 \\
(1,4-3,9) \\
4,2\end{array}$ & 0,001 \\
\hline $\begin{array}{l}\text { Padres que aprueban y tienen } \\
\text { actitudes permisivas hacia la AS en } \\
\text { la adolescencia }\end{array}$ & $99(22,1 \%)$ & 250 (50\%) & $(2,6-4,7)$ & $<0,001$ & $(2,8-6,3)$ & $<0,001$ \\
\hline
\end{tabular}

AS: Actividad sexual. ORc: Odds ratio crudo. ORa: Odds ratio ajustado. 
Tabla VI

ANÁLISIS BIVARIADO Y MULTIVARIADO, NIVEL SOCIOCULTURAL

\begin{tabular}{|c|c|c|c|c|c|c|}
\hline Nivel sociocultural & $\begin{array}{l}\operatorname{Sin} A S \\
(n: 447)\end{array}$ & $\begin{array}{l}\text { Con AS } \\
(\mathrm{n}: 500)\end{array}$ & $\begin{array}{c}\text { ORc } \\
\text { (IC 95\%) }\end{array}$ & $p$ & $\begin{array}{c}\text { ORa } \\
\text { (IC 95\%) }\end{array}$ & $\mathrm{p}$ \\
\hline $\begin{array}{l}\text { Estrato socioeconómico } \\
1 \text { y } 2 \text { vs } 3 \text { y más }\end{array}$ & $\begin{array}{c}283 \\
(63,3 \%)\end{array}$ & $\begin{array}{c}364 \\
(72,8 \%)\end{array}$ & $\begin{array}{c}1,6 \\
(1,2-2,1)\end{array}$ & 0,002 & $\begin{array}{c}1,5 \\
(1,1-2)\end{array}$ & 0,012 \\
\hline $\begin{array}{l}\text { Educación sexual por } \\
\text { personas diferentes }\end{array}$ & $\begin{array}{c}426 \\
(95,3 \%)\end{array}$ & $470(94 \%)$ & $\begin{array}{c}0,8 \\
(0,4-1,4)\end{array}$ & 0,376 & - & - \\
\hline
\end{tabular}

a padres (educadores y

personal de salud)

Haber recibido educa-

ción en planificación

familiar por personas

diferentes a padres

(educadores y personal

de salud)

\section{Pares o amigos que} aprueban y tienen actitu-

des permisivas hacia la

AS en la adolescencia

$\begin{array}{ccccc}403 & 429 & 0,7 & 0,004 & 0,6 \\ (90,1 \%) & (85,8 \%) & (0,4-1) & & (0,4-0,9)\end{array}$

389

(87\%)
489

$(97,8 \%)$
6,6

$(3,4-14,2)$
5,9

$(2,9-11,8)$
Son muchos los factores que se han asociado con el inicio de la actividad sexual temprana y embarazo en adolescentes. Las familias de un solo padre, bajo nivel educativo y de bajos ingresos, pobre supervisión de los padres y mensajes ambiguos de los adultos frente a la actividad sexual de los hijos, se han relacionado con una sexualidad precoz y una mayor frecuencia de embarazos. Paralelamente, la pobreza, el bajo nivel educativo y la baja cobertura de servicios de salud, fueron identificados como los principales factores de riesgo para practicar un aborto inducido en Colombia (14).

Aunque para nosotros la televisión y el internet no fueron factores de riesgo para el inicio de la AS temprana, se conoce de la influencia negativa que pueden tener estos por la cantidad de contenido sexual que tienen los programas a horas familiares, además de la publicidad, la música y material en internet y televisión con información sexual explícita. Y al respecto lo más grave es que la "supervisión de los padres" se ha perdido, ya que estos permanecen largas jornadas fuera de su casa. Cómo alternativa, los padres pueden ser capaces de reducir los efectos del contenido sexual al ver la televisión con sus hijos y discutir sus propias creencias sobre el sexo $(15,16)$.

En el análisis de este trabajo los factores que pudieran ser riesgo o protección para el inicio de la actividad sexual en la adolescencia se dividieron en tres niveles: el nivel individual, el nivel familiar y el nivel socio-cultural de las familias y los adolescentes.

En nuestro trabajo demostramos una asociación entre inicio de la AS y sexo masculino. Se han encontrado diferencias significativas en cuanto al sexo. El sexo masculino ha sido descrito como el más vulnerable para el inicio más temprano de la AS. En diferentes estudios, se encontró que las mujeres se perciben significativamente más seguras que los hombres de su habilidad para abstenerse de tener relaciones sexuales en situaciones en las que podrían sentirse inclinadas a hacerlo $(6,17)$.

Igualmente, encontramos que la aprobación por parte del adolescente de relaciones sexuales antes de los 20 años de edad se asoció significativamente con AS en la adolescencia. La aprobación de las relaciones sexuales por parte de los adolescentes se ve influenciada por su ambiente y contexto social. Hay trabajos que informan que el inicio de la actividad sexual está determinado por un número reducido de factores que involucran la influencia del contexto social y la percepción que tienen los adolescentes de la presión existente por parte de sus padres y de sus amigos para tener o no relaciones sexuales a su edad. Es importante señalar que la actitud personal y las variables de la norma social 
percibida se asocian positiva y significativamente entre sí (11).

En el presente estudio, encontramos un alto consumo de licor por parte de los adolescentes $(80,1 \%$ de todos los adolescentes; $68,8 \%$ en los que no han iniciado la AS y $89,7 \%$ en los que ya han iniciado la AS), cifra mucho más elevada que la informada por Grunbaum y cols (18), para quienes esta fue de $50 \%$. En Colombia el consumo de alcohol y sustancias psicoactivas ha aumentado, siendo las de mayor prevalencia el alcohol $(74,8 \%)$, tabaco $(37,7 \%)$, marihuana $(8,9 \%)$, cocaína $(4,5 \%)$ y éxtasis $(2,2 \%)$, cifras muy similares a las halladas en nuestros adolescentes (14). Además de la alta prevalencia de consumo de licor, el consumo de alcohol, tabaco y drogas psicoactivas mostraron una fuerte asociación con el inicio de la actividad sexual. Como es reconocido, el consumo de alcohol está relacionado a conductas de alto riesgo (19). Un adolescente que consume bebidas alcohólicas tiene mayor riesgo de iniciar su actividad sexual, que otra que no está expuesta a este hábito. Esta fuerte asociación ha sido informada en estudios previos (19-27) y la explicación más aceptada establece que además de ser un factor de riesgo asociado, es un factor causal (20). La Encuesta Nacional de Demografía y Salud ENDS 2010, informa que el 3\% de las jóvenes de 15 a 24 años de edad tuvo AS en el último año bajo efectos de alcohol y el $5 \%$ lo hizo en estado de embriaguez con una pareja que también lo estaba (9).

En el nivel familiar, se encontró que el no vivir con los padres, el compartir poco o ningún tiempo con la madre o el padre, el no recibir educación sexual por parte de la madre, el tener en casa más de dos hermanos, el que alguno de sus hermanos haya sido madre o padre en su adolescencia y el que sus padres aprueben y tengan actitudes permisivas hacia el sexo en la adolescencia, se hallaron asociados a inicio temprano de la AS. Sin embargo, después del análisis multivariado, sólo las variables, la madre no da educación sexual a su hijo, hermano/a que en la adolescencia haya sido madre o padre y el que sus padres aprueben y tengan actitudes permisivas hacia el sexo en la adolescencia, mantuvieron su asociación significativa con AS.

Respecto a los factores familiares, hay trabajos que señalan que los adolescentes que viven con los padres biológicos, tienen menor probabilidad de iniciar la AS de manera temprana, cuando se compara con aquellos que viven con un solo padre o con familias reconstituidas (28-30). El control y supervisión de los padres es un aspecto del ambiente familiar que se relaciona con la sexualidad de los adolescentes. Los hijos de padres que ejercen mayor control y supervisión, estableciendo límites y reglas a cerca de las actividades de noviazgo, presentan mayor probabilidad para retardar el inicio de la AS (31). Sin embargo, otros autores señalan que las conductas disciplinarias extremistas (déficit o exceso de normas) incrementan la probabilidad de inicio de AS entre adolescentes $(32,33)$. Caffery $(32)$, halló que, una relación nula o pobre entre el adolescente y sus padres, el primero puede presentar conductas de riesgo como sexo sin protección y se involucre en conductas sexuales como respuesta a su exceso de control y excesivo monitoreo por parte de sus padres. Por otro lado, en cuanto a la comunicación sobre temas de sexualidad, se reporta que cuando los padres discuten abiertamente con sus hijos, retrasan el inicio de la AS y cuando estos la inician, utilizan en mayor y mejor proporción métodos anticonceptivos $(34,35)$. Para nuestros adolescentes la madre tuvo mayor influencia en el retraso del inicio de la AS en adolescentes que el padre. Otros trabajos, han encontrado que los varones que mantienen una buena comunicación con su madre sobre temas de sexualidad, tienen una actividad sexual tardía. Sin embargo, la comunicación con el padre, por el contrario estuvo asociada a una AS más precoz, pero las mujeres que tenían una comunicación en estos temas con su padre, tienen una menor actividad sexual (10). En nuestro trabajo la escolaridad y ocupación de los padres no se halló asociado al inicio temprano de la AS, sin embargo, Andrade y cols (10), encontraron que la mayor escolaridad de los padres (bachillerato, carrera técnica o profesional) y el hecho de que el padre o la madre trabajen, son factores de riesgo para el inicio temprano de la AS.

En cuanto al nivel socio-cultural, los estratos socioeconómicos más deprimidos (estratos 1 y 2), fueron los que tuvieron mayor asociación con AS temprana en adolescentes. De igual manera, cuando los pares y amigos están de acuerdo con la AS en la adolescencia, esto influencia de manera positiva el inicio temprano de las relaciones sexuales entre los adolescentes. La información sobre planificación familiar impartida en colegios y por personal de salud u otros diferentes a los padres, tuvo una influencia protectora en el inicio de la actividad sexual. El nivel socio-económico tiene una asociación univariada fuerte con la iniciación temprana de la AS $(37,38)$. La mayoría de los estudios multivariados longitudinales coinciden en resaltar que el estado socioeconómico tiene muy poco efecto independiente sobre el momento del inicio de la AS (38). Estos resultados son alentadores, ya que sugieren que las intervenciones sobre las influencias del inicio de la AS temprana son eficaces. El comportamiento de los compañeros, pares o amigos, puede tener un efecto fuerte en la decisión de un adolescente en torno al inicio de la AS, donde la presión social puede tener un impacto más activo. La presión ejercida por compañeros (del mismo sexo o del sexo opuesto) puede resultar en un riesgo elevado de aparición de la AS temprana (39). No obstante, el apoyo mutuo para no tener 
sexo, es un factor protector para el inicio temprano de la AS (40). Brendgen y cols (41), hallaron que el rechazo de los pares se asocia indirectamente con un riesgo más elevado de AS temprana por su vinculación con la baja autoestima, pero sólo para las niñas. Mientras tanto, el abuso verbal por los profesores durante la infancia se asoció directamente con un mayor riesgo de tener relaciones sexuales tempranas.

Para entender la sexualidad del adolescente, podríamos verlo desde el concepto de interacción de Imogen King (42), mirando al adolescente como una persona social, con enorme capacidad de percibir y de tomar sus propias decisiones. A su edad el concepto de auto-cuidado aún se encuentra en construcción, sin embargo, este es participe en la toma de decisiones que afectan su vida y su salud. Los adolescentes tienen tres necesidades de salud fundamentales en los temas de la sexualidad, información útil y oportuna, cuidados para prevenir enfermedades y ayuda cuando las demandas de auto-cuidado no son satisfechas.

En el presente trabajo hemos evaluado los factores que se asocian con la AS del adolescente como un sistema abierto, compuesto por tres niveles: El individual, el familiar y el sociocultural. En el nivel individual, evaluamos al adolescente como una unidad total, como un yo individual. En este nivel encontramos que, los varones tienen una AS más temprana y prevalente que las mujeres, que el concepto propio a cerca de la sexualidad juega un papel en la toma de decisiones y que el consumo de alcohol, tabaco y sustancias psicoactivas precipitan el inicio de la AS. En este nivel, se ven comprometidos la autoimagen y el efecto de las influencias de amigos y del medio social. A nivel familiar se deja al descubierto las relaciones al interior de cada hogar, entre hijos, padres y hermanos, donde la madre juega un papel importante frente a la sexualidad del adolescente. El nivel socio-cultural es el que proporciona las estructuras para la interacción social, donde se definen las reglas de comportamiento, los modos de acción, las actitudes, valores y costumbres que se transmiten culturalmente a través de sistemas sociales tales como la familia o la escuela. En este nivel encontramos que los adolescentes de las clases menos favorecidas y la influencia de pares o grupo de amigos del adolescente, juegan un papel determinante del inicio de la AS en la adolescencia.

Las limitaciones del estudio fue que no incluimos a colegios privados de la ciudad, donde quizás otros factores pudieran jugar un papel importante, como es el familiar y social, ya que ellos están quizás pertenezcan a familias con mejores recursos económicos y mayor estrato socio-cultural. Esto puede afectar en cierta forma la validez externa de este trabajo a esa población.

\section{CONCLUSIÓN}

Factores individuales como sexo masculino, propia toma de decisiones, papel que desempeña la madre en la educación sexual de sus hijos y la influencia de pares o amigos en la toma de decisiones, se asociaron con el inicio temprano de la AS entre adolescentes.

\section{REFERENCIAS}

1. Trave T, Maya M, Vilaplana E. Repercusión neonatal del embarazo en adolescentes An Esp Pediatr 1989;3L(4):368-71.

2. Marchetti R, Rodríguez G, Rodríguez A. Los adolescentes y el SIDA: La importancia de la educación para la prevención. SIDA-FTS/1996;2(2):61-9.

3. Mendoza LA, Arias M, Pedroza M, Micolta P, Ramírez A, Cáceres Ch, López D, Núñez A, Acuña M. Actividad sexual en adolescencia temprana: problema de salud pública en una ciudad colombiana. Rev Chil Obstet Ginecol 2012;77(4):271-9.

4. Cutié JR, Laffita A, Toledo M. Primera relación sexual en adolescentes cubanos. Rev Chil Obstet Ginecol 2005;70(2):83-6.

5. Gillmore MR, Archibald ME, Morrison DM, Wilsdon A, Wells EA, Hoppe M, et al. Teen Sexual Behavior: Applicability of the Theory of Reasoned Action. J Marriage Family 2002;64(4):885-98.

6. Carvajal SC, Parcel GS, Banspach SW, BasenEngquist K, Coyle KK, Kirby D, et al. Psychosocial predictors of delay of first sexual intercourse by adolescents. Health Psychology 1999;18:443-52.

7. Pedersen W, Samuelsen SO, Wichstrøm L. Intercourse debut age: Poor resources, problem behavior or romantic appeal? J Sex Res 2003;40(4):333-45.

8. Profamilia, Bienestar Familiar, United States Agency for International Development, United Nations Population Fund. Salud sexual y reproductiva en Colombia, Encuesta Nacional de Demografía y Salud 2005. Bogotá: Profamilia, Bienestar Familiar, United States Agency International Development, United Nations Population Fund; 2005. Disponible en: http://www. profamilia.org.co/encuestas/Profamilia/Profamilia/index.php?option=com_content $\&$ view $=$ article $\& i d=62 \& \mathrm{lt}$ emid=9.

9. Profamilia, Ministerio de la Protección Social República de Colombia, Bienestar Familiar y United States Agency International Development, USAID del Pueblo de los estados Unidos. Conocimientos del VIH y otras ETS, Encuesta Nacional de Demografía y salud ENDS 2010. Informe de prensa, 2010. Disponible en: http://www.profamilia.org.co/encuestas/Profamilia/ Profamilia/index.php?option=com_content\&view=arti cle\&id $=62 \&$ Itemid $=9$.

10. Andrade P, Betancourt D, Palacios JR. Factores familiares asociados a la conducta sexual en adolescentes. Rev Colomb Psicol 2006;15:91-101. 
11. Vargas E, Barrera F. ¿Es la autoestima una variable relevante para los programas de prevención del inicio temprano de la actividad sexual? Acta Colomb Psicol 2005;13:133-61.

12. Silber TJ, Giurgiovich A, Munist MM. El embarazo en la adolescencia. En: Organización Panamericana de la Salud. La salud del adolescente y del joven. Washington, D.C.: OPS; 1995; p 252-63.

13. Mendoza LA, Arias MD, Mendoza LI. Hijo de madre adolescente: riesgos, morbilidad y mortalidad neonatal. Rev Chil Obstet Ginecol 2012;77(5):375-82.

14. Alba LE. Salud de la adolescencia en Colombia: bases para una medicina de prevención. Univ Méd Bogotá (Colombia) 2010;51(1):29-42.

15. Collins RL, Elliott MN, Berry SH, Kanouse DE, Kunkel $\mathrm{D}$, Hunter SB, et al. Watching sex on television predicts adolescent initiation of sexual behavior. Pediatrics 2004;114(3):e280-e9.

16. Ashby SL, Arcari CM, Edmonson MB. Television viewing and risk of sexual initiation by young adolescents. Arch Pediatr Adol Med 2006;160(4):375-80.

17. Rosenthal D, Moore S, Flynn I. Adolescent self-efficacy, self-esteem and sexual risk-taking. J Community \& Applied Social Psychol 1991;1:77-88.

18. Grunbaum J, Kann L, Kinchen S, Williams B, Ross J, Lowry $\mathrm{R}$, et al. Youth risk behavior surveillance United States, 2001. MMWR 2002;51(SS-4):1-64.

19. AAP Committee on Substance Abuse. Alcohol use and abuse: a pediatric concern. Pediatrics 1995;95:439-42.

20. Kirby D. Emerging answers: Research findings on programs to reduce teen pregnancy. Washington, DC: National Campaign to Prevent Teen Pregnancy; 2001:13-72

21. Kinsman S, Romer D, Furstenberg F, Schwarz D. Early sexual initiation: the role of peer norms. Pediatrics 1998;102:1185-92.

22. Hingson R, Heeren T, Winter M, Wechsler H. Early age of first drunkenness as a factor in college students' unplanned and unprotected sex attributable to drinking. Pediatrics 2003;111:34-41.

23. Dunn M, Bartee R, Perko M. Self-reported alcohol use and sexual behaviors of adolescents. Psychol Rep 2003;92:339-48.

24. Strasburger V. Alcohol advertising and adolescents. Pediatr Clin North Am 2002;49:353-76.

25. Strasburger V. Getting teenagers to say no to sex, drugs, and violence in the new millennium. Med Clin North Am 2000;84:787-810.

26. Brown R. Risk factors for substance abuse in adolescents. Pediatr Clin North Am 2002;49:247-55.

27. Ree D, Argys L, Averett S. New evidence on the relationship between substance use and adolescent sexual behavior. J Health Econ 2001;20:835-45.

28. Brewester KL, Billy JOG, Grady WR. Social context and adolescent behavior: The impact of community on the transition to sexual activity. Social Forces 1993;71:713-40.

29. Capaldi DM, Crosby L, Stoolmiller M. Predicting the timing of first sexual intercourse for at risk adolescent males. Child Development 1996;67:344-59.

30. Forste RT, Heaton TB. Initiation of sexual activity among female adolescent. Youth Soc 1998;19:250-68.

31. Hovell M, Sipan C, Blumberg E, Atkins C, Hofstetter $\mathrm{RC}$, Kreitner S. Family influences on Latino and Anglo adolescent's sexual behavior. J Marriage Fam 1994;53:973-986.

32. Caffery TE. A study on the effects of family functioning on attachment security in college students. Dissertation Abstracts International: section B: the sciences and engineering. 2000;60(10-B).

33. Upchurch DM, Aneshensel CS, Sucoff CA, LevyStorms L. Neighborhood and family contexts of adolescent sexual activity. J Marriage Fam 1999;61:920-33.

34. Fisher TD. Parent-child communication about sex and young adolescent's sexual knowledge and attitudes. Adolescence 1986;21:517-27.

35. Fox GL, Inazu JK. Patterns and outcomes of motherdaughter communication about sexuality. J Soc Issues 1980;36:7-29.

36. Andrade PP, Camacho VM, Díaz-Loving R. Comunicación con los padres y su relación con conducta sexual y percepción de riesgo de contraer SIDA. La psicología social en México 1992;4:102-7.

37. Kirby D. Antecedents of adolescent initiation of sex, contraceptive use, and pregnancy. Am J Health Behavior 2002;26(6):473-85.

38. Zimmer-Gembeck MJ, Helfand M. Ten years of longitudinal research on U.S. adolescent sexual behavior: developmental correlates of sexual intercourse, and the importance of age, gender and ethnic background. Developmental Rev 2008;28(2):153-224.

39. Laflin MT, Wang J, Barry M. A longitudinal study of adolescent transition from virgin to nonvirgin status. J Adol Health 2008;42(3):228-36.

40. Robinson G. Families, generations and self: Conflict, loyalty and recognition in an Australian aboriginal society. Ethos 1997;25(3):303-32.

41. Brendgen M, Wanner B, Vitaro F. Peer and teacher effects on the early onset of sexual intercourse. Am J Public Health 2007;97:2070-5.

42. Williams LA. Imogene King's interacting systems theory: Application in emergency and rural nursing. J Rural Nursing Health Care 2001;2(1):25-30. 